\title{
Soft Analysis of Discreet System Function Introducing Recursive Method
}

\author{
Barun Das \\ Astt. Professor,CSE \\ Dept. \\ Kanad Institute of \\ Engg. \& \\ Mgmt,Mankar,W.B,In \\ dia
}

\author{
Soumyendu \\ Bhattacharjee \\ HOD,ECE Dept. \\ W.B,India \\ Kanad Institute of \\ Engg. \& \\ Mgmt,Mankar,W.B, \\ India
}

\author{
Aniruddha Ghosh \\ Faculty, ECE Dept. \\ JIS College of \\ Engineering \\ Kalyani, Nadia, W.B
}

\author{
Biswarup \\ Neogi,Ph.D \\ Faculty, ECE Dept. \\ JIS College of \\ Engineering \\ Kalyani, Nadia, W.B., \\ India
}

\begin{abstract}
A major advantage of discrete domain analysis is the reduction of characteristic roots from infinite to finite numbers and consequent simplicity in the analysis and design of the controller. In this paper a new algorithm is proposed towards the sample data control and also analysis the time complexity is find out. The algorithm has order of $\mathrm{n} 2$ time complexity.
\end{abstract}

\section{Keywords}

Sample Data Control, Time Complexity, Space Complexity, Discreet Domain System, Recursive Method

\section{INTRODUCTION}

A system in which the signal appears at one or more points in the system as a sequence of pulses or numbers usually equally spaced in time. A type of digital control system in which one or more of the input or output signals is a continuous, or analog, signal that has been sampled. There are two type of sampled signal: sampling in time and quantization in amplitude. Sampling refers to the process of converting an analog signal from a continuously valued range of amplitude values to one of a finite set of possible numerical values [1]. The sampled-data systems have received significant attention to describe nonlinear system characteristics [2].A major advantage of discrete domain analysis is the reduction of characteristic roots from infinite to finite numbers and consequent simplicity in the analysis and design of the controller[3]. Discrete time control theory comes up with unusual, but useful, control designs, with negative PID tuning parameters being one of them[4].

\section{SAMPLED-DATA CONTROL REPRESENTATION WITH \\ POL YNOMIAL}

The sampled-data systems are used to describe nonlinear system characteristics. The discretization of a properly tuned continuous stable system expresses the preliminary approaches of nonlinear modeling. Considering the input test response as a step function, the generalized transfer function in Control System for sampled data control is represented as $[5,6,7]$

$$
\mathrm{T}(\mathrm{z})=\frac{\mathrm{C}(\mathrm{z})}{\mathrm{R}(\mathrm{z})}=\frac{\mathrm{A}_{0} \mathrm{z}^{0}+\mathrm{A}_{1} \mathrm{z}^{-1}+\mathrm{A}_{2} \mathrm{z}^{-2}+\ldots+\mathrm{A}_{\mathrm{n}} \mathrm{z}^{-\mathrm{n}}}{\mathrm{B}_{0} \mathrm{z}^{0}+\mathrm{B}_{1} \mathrm{z}^{-1}+\mathrm{B}_{2} \mathrm{z}^{-2}+\ldots .+\mathrm{B}_{\mathrm{m}} \mathrm{z}^{-\mathrm{m}}}
$$

Here, $\mathrm{R}(\mathrm{z})$ is the input function and $\mathrm{C}(\mathrm{z})$ is the output function.

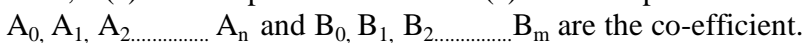
As the polynomial is in discrete domain, $\mathrm{z}=\mathrm{k}$ is considered

$$
\begin{aligned}
\mathrm{C}(\mathrm{k}) \mathrm{B}_{0}+\mathrm{C}(\mathrm{k}-1) & \mathrm{B}_{1}+\mathrm{C}(\mathrm{k}-2) \mathrm{B}_{2}+\cdots+\mathrm{C}(\mathrm{k}-\mathrm{m}) \mathrm{B}_{\mathrm{m}} \\
= & {\left[\left\{\mathrm{R}(\mathrm{k}) \mathrm{A}_{0}+\mathrm{R}(\mathrm{k}-1) \mathrm{A}_{1}+\mathrm{R}(\mathrm{k}-2) \mathrm{A}_{2}\right.\right.} \\
& \left.\left.+\cdots+\mathrm{R}(\mathrm{k}-\mathrm{n}) \mathrm{A}_{\mathrm{n}}\right\}\right]
\end{aligned}
$$

Where $\mathrm{k}=0,1,2,3, \ldots$.

$$
\begin{gathered}
\mathrm{C}(\mathrm{k})=\frac{1}{B_{0}}\left[\left\{\mathrm{R}(\mathrm{k}) \mathrm{A}_{0}+\mathrm{R}(\mathrm{k}-1) \mathrm{A}_{1}+\mathrm{R}(\mathrm{k}-2) \mathrm{A}_{2}+\ldots+\right.\right. \\
\left.\mathrm{R}(\mathrm{k}-\mathrm{n}) \mathrm{A}_{\mathrm{n}}\right\}-\mathrm{C}(\mathrm{k}-1) \mathrm{B}_{1} \mathrm{C}(\mathrm{k}-2) \mathrm{B}_{2}+ \\
\left.\left.\ldots+\mathrm{C}(\mathrm{k}-\mathrm{m}) \mathrm{B}_{\mathrm{m}}\right\}\right] \text { Applying }
\end{gathered}
$$

technique on the transfer function $\mathrm{T}(\mathrm{z})$

$$
\mathrm{T}(\mathrm{z})=\frac{1.213 z^{-1}+0.01471 z^{-2}+0.07518 z^{-3}-0.004608 z^{-4}}{z^{0}+0.2121 z^{-1}+0.08263 z^{-2}+0.003374 z^{-3}+0.0005531 z^{-4}}
$$

Coefficients of equation are:

$$
\begin{aligned}
& \mathrm{A}_{0}=0, \mathrm{~A}_{1}=1.213, \mathrm{~A}_{2}=0.01471, \mathrm{~A}_{3}=0.0751, \mathrm{~A}_{4}=0.004608 \\
& \mathrm{~B}_{0}=1, \mathrm{~B}_{1}=0.2121, \mathrm{~B}_{2}=0.0826, \mathrm{~B}_{3}=0.00337, \mathrm{~B}_{4}=0.0005531
\end{aligned}
$$

Now for unit step input $\mathrm{R}(0)=\mathrm{R}(1)=\mathrm{R}(2)=\ldots . .=\mathrm{R}(\mathrm{k})=1$. So considering $\mathrm{k}=0,1,2,3, \ldots ., 10$, the values of $\mathrm{C}(0) \mathrm{C}(1) \mathrm{C}(2) \mathrm{C}(3) \mathrm{C}(4) \ldots \ldots \ldots . . . \mathrm{C}(10)$ are obtained as shown in the table 1.[8] 
Table1. Table for values of $c(k)$ for different values of $k$

\begin{tabular}{|c|c|}
\hline $\mathrm{C}(\mathrm{k})$ & Generalized Expression \\
\hline $\mathrm{C}(0)$ & $\mathrm{C}(0)=\frac{1}{\mathrm{~B}_{0}}\left[\mathrm{~A}_{0} \mathrm{R}(0)\right]$ \\
\hline $\mathrm{C}(1)$ & $\frac{1}{B_{0}}\left[\left\{A_{0} R(1)+A_{1} R(0)\right\}-C(0) B(1)\right]$ \\
\hline $\mathrm{C}(2)$ & $\begin{array}{r}\frac{1}{B_{0}}\left[\left\{A_{0} R(2)+A_{1} R(1)+A_{2} R(0)\right\}\right. \\
\left.-\left\{C(1) B_{1}+C(0) B_{2}\right\}\right]\end{array}$ \\
\hline $\mathrm{C}(3)$ & $\begin{aligned} \frac{1}{\mathrm{~B}_{0}}\left[\left\{\mathrm{~A}_{0} \mathrm{R}(3)+\mathrm{A}_{1} \mathrm{R}(2)+\mathrm{A}_{2} \mathrm{R}(1)+\mathrm{A}_{3} \mathrm{R}(0)\right\}\right. & \\
& \left.-\left\{\mathrm{C}(2) \mathrm{B}_{1}+\mathrm{C}(1) \mathrm{B}_{2}+\mathrm{C}(0) \mathrm{B}_{3}\right\}\right]\end{aligned}$ \\
\hline $\mathrm{C}(4)$ & $\begin{array}{c}\frac{1}{\mathrm{~B}_{0}}\left[\left\{\mathrm{~A}_{0} \mathrm{R}(4)+\mathrm{A}_{1} \mathrm{R}(3)+\mathrm{A}_{2} \mathrm{R}(2)+\mathrm{A}_{3} \mathrm{R}(1)+\mathrm{A}_{4} \mathrm{R}(0)\right\}\right. \\
\left.-\left\{\mathrm{C}(3) \mathrm{B}_{1}+\mathrm{C}(2) \mathrm{B}_{2}+\mathrm{C}(1) B_{1}+\mathrm{C}(0) \mathrm{B}_{4}\right\}\right]\end{array}$ \\
\hline $\mathrm{C}(5)$ & $\begin{array}{c}\frac{1}{\mathrm{~B}_{0}}\left[\left\{\mathrm{~A}_{0} \mathrm{R}(5)+\mathrm{A}_{1} \mathrm{R}(4)+\mathrm{A}_{2} \mathrm{R}(3)+\mathrm{A}_{3} \mathrm{R}(2)+\mathrm{A}_{4} \mathrm{R}(1)\right.\right. \\
\left.+\mathrm{A}_{5} \mathrm{R}(0)\right\} \\
\left.-\left\{\mathrm{C}(4) \mathrm{B}_{1}+\mathrm{C}(3) \mathrm{B}_{2}+\mathrm{C}(2) \mathrm{B}_{3}+\mathrm{C}(1) \mathrm{B}_{4}+\mathrm{C}(0) \mathrm{B}_{5}\right\}\right]\end{array}$ \\
\hline$C(6)$ & $\begin{array}{c}\frac{1}{\mathrm{~B}_{0}}\left[\left\{\mathrm{~A}_{0} \mathrm{R}(6)+\mathrm{A}_{1} \mathrm{R}(5)+\mathrm{A}_{2} \mathrm{R}(4)+\mathrm{A}_{3} \mathrm{R}(3)+\mathrm{A}_{4} \mathrm{R}(2)\right.\right. \\
\left.+\mathrm{A}_{5} \mathrm{R}(1)+A_{6} R(0)\right\} \\
-\left\{\mathrm{C}(5) \mathrm{B}_{1}+\mathrm{C}(4) \mathrm{B}_{2}+\mathrm{C}(3) \mathrm{B}_{3}+\mathrm{C}(2) \mathrm{B}_{4}+\mathrm{C}(1) \mathrm{B}_{5}\right. \\
\left.\left.+C(0) B_{6}\right\}\right]\end{array}$ \\
\hline $\mathrm{C}(7)$ & $\begin{array}{r}\frac{1}{\mathrm{~B}_{0}}\left[\left\{\mathrm{~A}_{0} \mathrm{R}(7)+\mathrm{A}_{1} \mathrm{R}(6)+\mathrm{A}_{2} \mathrm{R}(5)+\mathrm{A}_{3} \mathrm{R}(4)+\mathrm{A}_{4} \mathrm{R}(3)\right.\right. \\
+\mathrm{A}_{5} \mathrm{R}(2)+A_{6} R(1) \\
\left.+A_{7} R(0)\right\}-\left\{\mathrm{C}(6) \mathrm{B}_{1}+\mathrm{C}(5) \mathrm{B}_{2}+\mathrm{C}(4) \mathrm{B}_{3}+\mathrm{C}(3) \mathrm{B}_{4}\right. \\
\left.\left.+\mathrm{C}(2) \mathrm{B}_{5}+C(1) B_{6}+C(0) B_{7}\right\}\right]\end{array}$ \\
\hline
\end{tabular}

\section{ALGORITHM APPROACH TOWARD THE SAMPLE DATA CONTROL SIMULATOR GENERATOR}

Algorithm: sample_datacontrol()

Step1: Start.

Step2: initialize the array A[] \& B[]. /*coefficient of A \& B are store in the array*/

Step3: initialize the variable sum \& sum1 with 0.0.

Step4: Read k /*for finding out put function $\mathrm{c}(\mathrm{k})$ of sample data control $* /$

Step5: for $\mathrm{i}=0$ to $\mathrm{k}$

do $\mathrm{R}(\mathrm{i})=1$

Step6: for $\mathrm{j}=0$ to $\mathrm{i}$

Step7: sum $=\operatorname{sum}+(A(j) * R(i-j))$

Step8: $\quad k=i-j$

Step9: if(i>0) goto step 5

Step10: else

Step11: sum1 $=$ sum $1+(\mathrm{C}(\mathrm{k}-1) * \mathrm{~B}(\mathrm{j}))$

Step12: $C(\mathrm{i})=1 / \mathrm{B}[0]^{*}($ sum-sum1 $)$

\section{FLOW CHART FOR SAMPLE DATA CONTROL SIMULATOR GENERATOR}

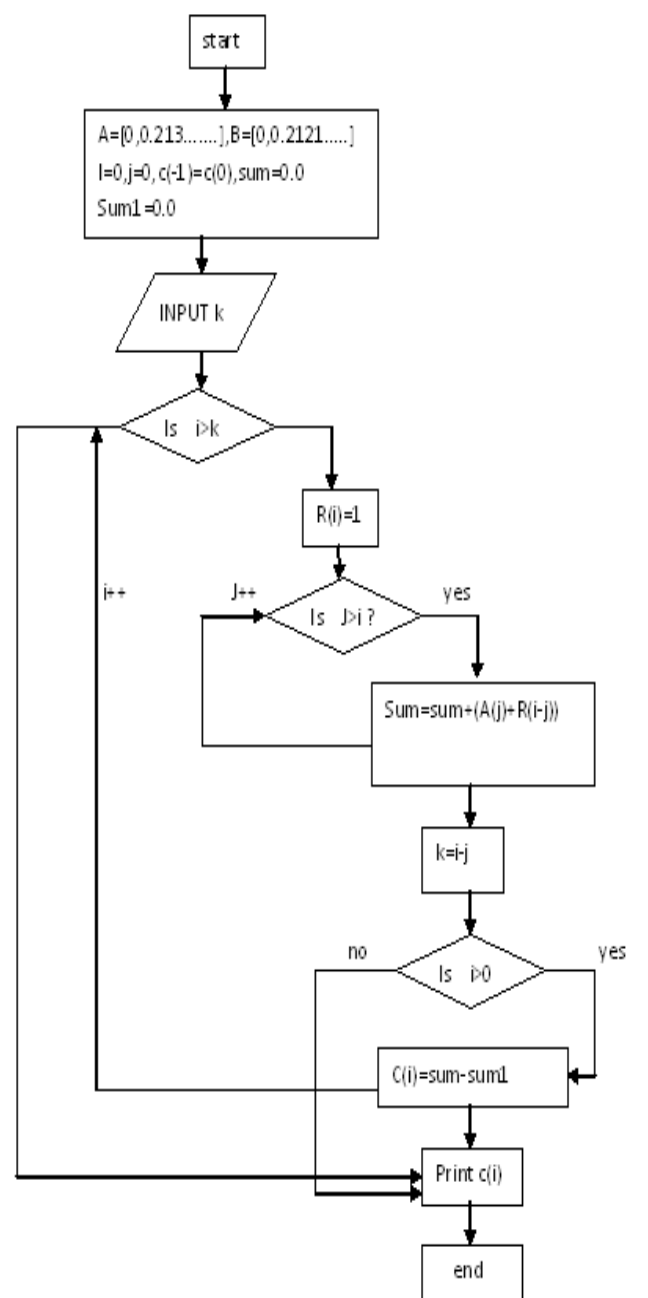

Fig 1: Flowchart for sample data control simulator generator

\section{TIME SPACE COMPLEXITY REPRESENTATION}

Time complexity of an algorithm T(n) is defined to be the time taken over all inputs of size $n$. The worst case time complexity is the maximum time taken. Space complexity of an algorithm $\mathrm{T}(\mathrm{n})$ is the amount of memory used over all inputs of size $\mathrm{n}$. The worst case or average case running time or memory usage of an algorithm is often expressed as a function of the length of its input using big $\mathrm{O}$ notation. By writing $\mathrm{T}(\mathrm{n})=$ $\mathrm{O}(\mathrm{n} 2)$, the algorithm has order of $\mathrm{n} 2$ time complexity. [1]Big$\mathrm{O}$ notation deals in upper bounds, technically speaking an algorithm is $\mathrm{O}(\mathrm{g}(\mathrm{n}))$ for any and all(n) that grow asymptotically faster than $f(n)$, so if an algorithm is $O(n)$ it must be $\mathrm{O}(\mathrm{n} 2)$ [2]. 
Table2. Table for cost and time for finding the complexity

\begin{tabular}{|l|c|l|}
\hline \multicolumn{1}{|c|}{ ALGO } & COST & TIME \\
\hline i)for $\mathrm{i}=0$ to $\mathrm{k}$ & $\mathrm{C}_{1}$ & $\mathrm{n}$ \\
\hline ii) $\mathrm{R}(\mathrm{i})=1$ & $\mathrm{C}_{2}$ & $\mathrm{n}$ \\
\hline iii) for $\mathrm{j}=0$ to $\mathrm{i}$ & $\mathrm{C}_{3}$ & $\sum_{j=0}^{n} t_{\mathrm{j}}$ \\
\hline iv) sum $=\operatorname{sum}+(\mathrm{A}(\mathrm{j}) * \mathrm{R}(\mathrm{i}-\mathrm{j}))$ & $\mathrm{C}_{4}$ & $\sum_{j=0}^{n} t_{\mathrm{j}}-1$ \\
\hline v) $\mathrm{k}=1-\mathrm{j}$ & & \\
\hline vi) $\operatorname{sum} 1=\operatorname{sum} 1+(\mathrm{C}(\mathrm{k}-1) * \mathrm{~B}(\mathrm{j})$ & $\mathrm{C} 6$ & $\sum_{j=0}^{n} t_{\mathrm{j}-1}$ \\
& & \\
\hline vii) $\mathrm{C}[\mathrm{i}]=\frac{1}{B[0]} *(\operatorname{sum}-\mathrm{sum} 1)$ & $\mathrm{C} 7$ & $\mathrm{n}$ \\
& & \\
\hline
\end{tabular}

The running time of the algorithm is the sum of running times for each statement executed; a statement that takes $\mathrm{Ci}$ step to execute and execute $\mathrm{n}$ times will contribute $\mathrm{Ci}{ }^{*} \mathrm{n}$ to the total running time. To compute $\mathrm{T}(\mathrm{n})$, the running time of this algorithm on an input of $n$ values, the sum of the product of the cost and time columns, obtaining

$$
\begin{aligned}
& \mathrm{T}(\mathrm{n})=\mathrm{C} 1 * \mathrm{n}+\mathrm{C} 2 * \mathrm{n}+\mathrm{C} 3 * \sum_{j=0}^{n} t \mathrm{j}+\mathrm{C} 4 * \sum_{j=0}^{n} t \mathrm{j}-1+\mathrm{C} 5 \\
& * \sum_{j=0}^{n} t \mathrm{j}-1+\mathrm{C} 6 * \sum_{j=0}^{n} t \mathrm{j}-1+\mathrm{C} 7 * \mathrm{n} \\
& \mathrm{T}(\mathrm{n})=\mathrm{C}_{1} * \mathrm{n}+\mathrm{C}_{2} * \mathrm{n}+\mathrm{C}_{3} * \frac{n(n+1)}{2}+\mathrm{C}_{4} * \frac{n^{2}+n-2}{2}+\mathrm{C}_{5} * \frac{n^{2}+n-2}{2} \\
& +\mathrm{C}_{6^{*}} \frac{n^{2}+n-2}{2}+\mathrm{C}_{7 *} \mathrm{n} \\
& \mathrm{T}(\mathrm{n})=\mathrm{C}_{1} \mathrm{n}+\mathrm{C}_{2} \mathrm{n}+\mathrm{C}_{7} \mathrm{n}+\mathrm{C}_{3} \frac{n(n+1)}{2}+\left(\mathrm{C}_{4}+\mathrm{C}_{5}+\mathrm{C}_{6}\right)\left(\frac{n(n+1)}{2}-\right. \\
& 1) \\
& \mathrm{T}(\mathrm{n})=\mathrm{C}_{1} \mathrm{n}+\mathrm{C}_{2} \mathrm{n}+\mathrm{C}_{7} \mathrm{n}+\frac{n(n+1)}{2}\left(\mathrm{C}_{3}+\mathrm{C}_{4}+\mathrm{C}_{5}+\mathrm{C}_{6}\right)-\left(\mathrm{C}_{4}+\mathrm{C}_{5}\right. \\
& \left.+\mathrm{C}_{6}\right) \\
& \mathrm{T}(\mathrm{n})=\left(\mathrm{C}_{1} \mathrm{n}+\mathrm{C}_{2} \mathrm{n}+\mathrm{C}_{7} \mathrm{n}\right)+\frac{n^{2}}{2}\left(\mathrm{C}_{3}+\mathrm{C}_{4}+\mathrm{C}_{5}+\mathrm{C}_{6}\right)+\frac{n}{2}\left(\mathrm{C}_{3}\right. \\
& \left.+\mathrm{C}_{4}+\mathrm{C}_{5}+\mathrm{C}_{6}\right)-\left(\mathrm{C}_{4}+\mathrm{C}_{5}+\mathrm{C}_{6}\right) \\
& \mathrm{T}(\mathrm{n})=\left(\mathrm{C}_{1}+\mathrm{C}_{2}+\mathrm{C}_{7}\right) \mathrm{n}+\frac{n}{2}\left(\mathrm{C}_{3}+\mathrm{C}_{4}+\mathrm{C}_{5}+\mathrm{C}_{6}\right)+\frac{n^{2}}{2}\left(\mathrm{C}_{3}+\mathrm{C}_{4}\right. \\
& \left.+\mathrm{C}_{5}+\mathrm{C}_{6}\right)-\left(\mathrm{C}_{4}+\mathrm{C}_{5}+\mathrm{C}_{6}\right) \\
& \mathrm{T}(\mathrm{n})=n^{2}\left(\frac{\mathrm{C}_{3}}{2}+\frac{C 4}{2}+\frac{C 5}{2}+\frac{C 6}{2}\right)+\mathrm{n}\left(\mathrm{C}_{1}+\mathrm{C}_{2}+\mathrm{C}_{7}+\frac{\mathrm{C} 3}{2}+\frac{C 4}{2}+\right. \\
& \left.\frac{C 5}{2}+\frac{C 6}{2}\right)-\left(\mathrm{C}_{4}+\mathrm{C}_{5}+\mathrm{C}_{6}\right)
\end{aligned}
$$

\section{CONCLUSION}

The analysis of sampled-data systems leads to new challenging control problems in today's researcher. The paper is an attempted to solve the discreet domain analysis very easily. As per the future concern of this work, the simulation software will be developed to support the sampled data control field. So the running time can be expressed as : $\mathrm{an}^{2}+\mathrm{bn}+\mathrm{c}$ Throwing the lower order term and ignoring the complexity can be written as $\mathrm{O}\left(\mathbf{n}^{2}\right)$.

\section{REFERENCES}

[1] Jonh R. Ragazzini,Gene F.Franklin: "SAMPLE-DATA CONTROL SYSTEMS"McGraw-hill book company,inc.NEW YORW,1958.

[2] Li-Sheng $\mathrm{Hu}$, Yong-Yan Cao, Hui-He Shao "Robust and Nonlinear Control" International Journal [ DOI: 10.1002/rnc.632] Volume 12, Issue 5, pages 447464, 30 April 2002.

[3] Ilia G. Polushin , Horacio J. Marquez"Multirate versions of sampled-data stabilization of nonlinear systems" science direct, accepted 16 January 2004,Canada.

[4] Okuyama, Y " Robust stabilization and PID control for nonlinear discretized systems on a grid pattern" American Control Conference, IEEE Sponsored,2008, Tokushima.

[5] E.I Jury, Theory and Application of z-Transformation Method New York: Willey; 1946.

[6] B.D.O Anderson, E.I.Jury and Mansour, "Schwarz matrix properties for Continues and discrete time systems,'Int.J.Contr,Vol.33, pp,1-16,1976.

[7] B.Neogi, R.Darbar, S.Mandal, B.Gorai, S.Ghosh, A. Das \&D.N.Tibarewala, "Optimal Design of Prosthetic Limb Control System with Paraplegia and Fatigue Condition" accepted in International Conference on Emerging Applications of Information Technology IEEE Sponsored CSI, Kolkata.

[8] Sw1ti Banerjee, Soumyendu Bhattacharjee, Avishek Nag, Sreya Bhattacharya, Dr. Biswarup Neogi” DISCRETE DOMAIN ANALYSIS OF DEXTEROUS

[9] HAND MODEL BY SIMULATION ASPECT" $2^{\text {nd }}$ International Conference On Computer, Communication,

[10] Control and Information Technolog,2011, science direct,Kolkata.

[11] Thomas H. Cormen, Charles E. Leiserson:” Introduction to Algorithms" McGraw-Hill Osborne, Second Edition.

[12] Dexter C. Kozen:" The Design and Analysis of Algorithms", Springer 